\title{
Small steps in thermonuclear supernova research
}

\section{Eduardo Bravo and Domingo García-Senz}

Department of Physics and Nuclear Engineering (UPC) and IEEC

\section{Inmaculada Domínguez}

Departamento de Física Teórica y del Cosmos, Univ. Granada

Type Ia supernovae are the result of the thermonuclear disruption of massive white dwarfs made of carbon and oxygen, but the details of the explosion mechanism are largely unknown. Most current explosion models invoke either a pure turbulent deflagration or a delayed transition to a supersonic detonation. Although both mechanisms can coexist, and account for part of the observational sample, only delayed detonations seem able to explain the bulk of the so-called normal Type Ia supernovae. Here we report on two numerical experiments aimed to explore the conditions under which a deflagration-to-detonation transition can be obtained, and their consequences

Supernovae: lights in the darkness

October 3-5, 2007

Mao (Menorca) 


\section{Introduction}

The knowledge of the physical mechanism by which a white dwarf is disrupted by a thermonuclear explosion is relevant to many topics of modern astrophysics. A satisfactory model of the explosion becomes crucial to better understand Type Ia supernovae, which in turn have profound implications in cosmology and in studies of the dynamics of the interstellar medium and the chemical evolution of galaxies. Although the more plausible models of the explosion always involve the thermonuclear disruption of a white dwarf, the current zoo of explosion mechanisms is still too large to be useful in cosmological applications of Type Ia supernovae or to make it possible to understand the details of the chemical evolution of the Galaxy. Nowadays, the favored SNIa model is the thermonuclear explosion of a white dwarf that approaches the Chandrasekhar-mass limit owing to accretion from a companion star at the appropriate rate to avoid the nova instability. Other types of models, such as the sub-Chandrasekhar models or the double degenerate scenario, although not completely ruled out, either are not able to explain even the gross features of the spectrum and light curve of normal supernovae, or face theoretical objections (e.g. Nugent et al. [1997], Napiwotzki et al. [2002], Segretain, Chabrier \& Mochkovitch [1997], Saio \& Nomoto [1998]).

The huge increase in number, quality and diversity of observational data related to Type Ia supernovae (SNIa) in recent years, combined with the advance in computer technology, have persuaded modelers to leave the phenomenological calculations that rely on spherical symmetry, and attempt more physically meaningful multidimensional simulations. Admitting that at least part of the diversity shown by the Type Ia supernovae sample is directly related to the explosion mechanism of a Chandrasekhar-mass white dwarf (Hatano et al. [2000]), current multidimensional hydrodynamical calculations should be able to allow for a wide range of nickel masses synthesized in different events. Lacking multidimensional studies of the light curve and spectra, it is certainly risky to interpret the results of the three dimensional explosion simulations in order to elucidate the explosion mechanism and discriminate between models. However, even admitting that a perfect match between the observed luminosity and spectral evolution of SNIa and those deduced on the base of angle-averaged versions of the 3D explosion models is not to be expected, there are a series of tests that a good normal SNIa model should pass:

- There should be no more than a few tenths of unburnt carbon moving at low velocities (Marion et al. [2003], Baron, Lentz \& Hauschildt [2003], Kozma et al. [2005]).

- The ejected mass of ${ }^{56} \mathrm{Ni}$ should reach $\sim 1 \mathrm{M}_{\odot}$, after neutronization due to electron captures is properly taken into account (Mazzali et al. [2007]).

- The final kinetic energy should be on the order or larger than 1 foe ( 1 foe $\equiv 10^{51}$ ergs).

- Intermediate-mass elements should be generated in amounts exceeding $\sim 0.2 \mathrm{M}_{\odot}$ (GarcíaSenz et al. [2007]).

- The ejecta should be chemically stratified, as demanded by observations of supernovae and remnants (Badenes et al.[2006], Gerardy et al.[2007], Mazzali et al. [2008]).

- Large clumps of radioactive ${ }^{56} \mathrm{Ni}$ and other Fe-group elements should not be present at the photosphere at the time of maximum brightness (Thomas et al. [2002], Baron et al. [2008]). 
Nowadays the best models involve a white dwarf with a mass near the Chandrasekhar-mass limit in which a nuclear fuel ignites in one or many sparks around the center and thermonuclear combustion propagates henceforth through the rest of the star. In these models the starting point of hydrodynamical calculations is the thermal runaway caused by the screened ${ }^{12} \mathrm{C}+{ }^{12} \mathrm{C}$ nuclear reaction in degenerate conditions. The shape of the initially incinerated region probably does not keep the spherical symmetry that characterizes previous, hydrostatic, stages due to the stochastic nature of ignition and to the role played by hydrodynamic instabilities. Many exploratory studies carried out in the past using one-dimensional hydrodynamics have shown that the key point to achieve a successful explosion relies in the proper estimation of the velocity of the combustion front (Niemeyer \& Woosley [1997], Hillebrandt \& Niemeyer [2000]). Although the flame velocity is large, close to $100 \mathrm{~km} \mathrm{~s}^{-1}$ (Timmes \& Woosley [1992]), it is quite subsonic: $\simeq 0.01 v_{\text {sound }}$, where $v_{\text {sound }}$ is the sound speed. These velocities are far below the nearly supersonic velocities which, according to many one-dimensional simulations, are needed to blow away the star. Accounting for turbulent acceleration of the flame can help obtaining an energetic explosion, but still far below what is needed to explain bright SNIa, even after an accurate selection of the optimal initial conditions at the thermal runaway (Röpke et al. [2007]).

There have been several attempts to bypass the weaknesses of deflagration models, preserving at the same time many of their advantages, by postulating a sudden transition from a subsonic deflagration to a supersonic detonation (Ivanova et al. [1974], Khokhlov [1991]), i.e. the multidimensional version of the delayed detonation model: Arnett \& Livne [1994], Livne [1999], Golombek \& Niemeyer [2005] in 2D; García-Senz \& Bravo [2003], Gamezo, Khokhlov, \& Oran [2004, 2005], Röpke \& Niemeyer [2007] in 3D. In particular, the recent work of Gamezo et al. [2005] produced a satisfactory thermonuclear explosion, thus solving many of the problems of pure deflagration models. The density at which the jump to the detonation takes place had to be estimated because the physics behind the deflagration-to-detonation (DDT) transition is not well understood. In Gamezo et al. [2005] the adopted transition density was rather high, $\rho_{\mathrm{t}} \geq 2.5 \times 10^{8} \mathrm{~g} \mathrm{~cm}^{-3}$ and the detonation was induced near the center of the white dwarf. Röpke \& Niemeyer [2007] explored the range of explosions that would result if the DDT took place in the outer shells, at $r \sim 1.7-1.8 \times 10^{8} \mathrm{~cm}$, after a huge expansion due to subsonic combustion initiated in a few hot bubbles. They used a physically motivated criterion to switch on a DDT, namely that the combustion entered the distributed regime, in which mixing of burnt and fresh fuel by turbulence fluctuations at the scale of the flame width might trigger a detonation. This criterion led to consistent results with respect to the final kinetic energy and to the mass of Fe-group and intermediate-mass elements synthesized. It also allowed the almost complete depletion of carbon in the center.

In this work we have explored the conditions under which a deflagration-to-detonation transition can arise within C-O matter contaminated by traces of He. The context of our work, the methodology, and the results are presented in the next Section. Later, we show the results from three-dimensional simulations of the delayed-detonation scenario aimed to discern the sensitivity of the explosion properties on the location of the region where the DDT occurs.

\section{The role of $\mathrm{He}$ in deflagration-to-detonation transition}

The essential feature of detonation initiation is the formation of a non-uniformly preheated 
region with a level of fluctuations of temperature, density, and chemical composition such that a sufficiently large mass burns before a sonic wave can cross it. The thermal gradient needed is given by (Khokhlov [1991]):

$$
\nabla T<\frac{\Theta T}{A v_{\text {sound }} \tau_{i}}
$$

where $A$ is a numerical coefficient, $A \sim 0.2-5.0, \tau_{i}=T / \dot{T}$ is the induction time at the temperature $T$, and $\Theta \sim 0.04-0.05$ is the Frank-Kameneetskii factor:

$$
\Theta=-\frac{\partial \ln \tau_{i}}{\partial \ln T}
$$

Such fluctuacions could be produced by a variety of mechanisms: adiabatic pre-compression in front of a deflagration wave, shock heating, mixing of hot ashes with fresh fuel (Khokhlov [1991]), accumulation of pressure waves due to a topologically complex geometrical structure of the flame front, or transition to the distributed burning regime (Niemeyer \& Woosley [1997]). Among the proposed mechanisms of transition, turbulence pre-conditioning has received the most attention. Khokhlov, Oran \& Wheeler [1997] determined the criteria for a DDT in unconfined conditions such as those realized during the expansion of a white dwarf following a deflagration phase. For a DDT to be feasible, the turbulent velocity has to exceed the laminar flame velocity by a factor 1-8 at a lengthscale comparable to the detonation wave thickness. This criteria was fulfilled for flame densities in the range $5 \times 10^{6} \mathrm{~g} / \mathrm{cm}^{3}<\rho<2-5 \times 10^{7} \mathrm{~g} / \mathrm{cm}^{3}$ for reasonable assumptions. At densities in excess of $10^{8} \mathrm{~g} / \mathrm{cm}^{3}$ a DDT transition is quite unlikely (Khokhlov et al. [1997], but see Zingale \& Dursi [2007] who pointed to bubbles fragmentation as a way to increase the flame surface and facilitate a DDT at $\rho \sim 2 \times 10^{8} \mathrm{~g} / \mathrm{cm}^{3}$ ).

Small-scale simulations are needed to ascertain if the necessary conditions for a DDT are actually reached during a white dwarf explosion driven by a deflagration wave. Up to the present, such studies seem to disfavor a DDT in view of the robustness of subsonic flames against interaction with vortical flow (Röpke, Hillebrandt \& Niemeyer [2004]) for the typical maximum expected velocites at the integral scale, $\sim 100 \mathrm{~km} / \mathrm{s}$ (Lisewski, Hillebrandt \& Woosley [2000]). Moreover, Niemeyer [1999] estimated that the size of the fluctuations that can be expected from turbulence at the critical densities is $\sim 3$ orders of magnitude smaller than required for a DDT to occur.

One way to favor a deflagration-to-detonation transition in C-O matter is to mix it with a small quantity of $\mathrm{He}$, which both is more reactive and releases more energy than carbon. Actually, a very small cap made of He might rest atop of the white dwarf at the moment of carbon runaway, as a result of previous accretion from the companion star in the binary system. This helium could either have been accreted directly from a He star or result from the nuclear processing of accreted hydrogen. Höflich \& Khokhlov [1996] suggested the pressence of a $\sim 0.01 \mathrm{M}_{\odot}$ cap of He would improve the match of calculated and observed light curves. This helium might accumulate from the surrounding accretion disk if the accretion rate were not constant, for instance if it dropped below the critical rate for steady burning. Cumming et al. [1994, 1994] detected indications of two He I lines at $2.04 \mu \mathrm{m}$ and $1.052 \mu \mathrm{m}$ in the spectra of the spectroscopically normal SN 1994D. The absorption feature at $1.05 \mu \mathrm{m}$ has been since detected in the early spectra of many other SNIa (see Nomoto et al. [2003], Pignata et al [2008]), although its identification is problematic since 
this line could rather be due to Mg II (Wheeler et al. [1998], Hatano et al. [1999]). An interesting possibility is that the $\mathrm{He}$ I line is blended with other lines from magnesium and, possibly, other intermediate-mass elements. Up to $\sim 0.01 \mathrm{M}_{\odot}$ of He might remain hidden this way (Mazzali \& Lucy [1998]).

If it actually exists, the small cap of helium will have several chances to mix with the outer layers of the surrounding $\mathrm{C}-\mathrm{O}$ rich white dwarf. One first opportunity arises during the convective simmering phase that affects most of the white dwarf shortly before the explosion (Khulen, Woosley \& Glatzmaier [2006], Piro \& Bildsten [2008], Chamulak et al. [2008]). Even though convection during simmering is not expected to reach the outermost layers of the white dwarf, the associated motion can excite pulsating and non-spherical mode instabilities of the star that would facilitate diffusion of $\mathrm{He}$ into the $\mathrm{C}-\mathrm{O}$ layers. Another opportunity for mixing He within $\mathrm{C}-\mathrm{O}$ layers is during the deflagration phase of the explosion itself. Turbulence and, especially, Rayleigh-Taylor instability induce large radial excursions of huge volumes of ashes that destabilize the whole white dwarf and can result once more in the mixing of an eventual external He layer with the C-O beneath it. Hence, even though it may seem a speculative scenario, one cannot discard the possibility that $\mathrm{He}$ is present in some small mass fraction within the matter that is prone to detonate in a DDT model. Mixing of He within a detonating region seems more likely in scenarios in which the transition to detonation occurs in the outermost layers of the white dwarf, e.g. those described in Plewa, Calder \& Lamb [2004], Bravo \& García-Senz [2006] and Röpke, Woosley \& Hillebrandt [2007].

We have explored the effect due to mixing of a small quantity of He with the predominant C-O material upon the conditions needed to launch a detonation in a white dwarf, following a methodology similar to that of Arnett \& Livne [1994] and Niemeyer \& Woosley [1997]. We have followed with a one-dimensional supernova code the hydrodynamical and nuclear evolution of a uniform density sphere made of $\mathrm{C}-\mathrm{O}$ in equal amounts containing a small mass fraction of He. This thermonuclear bomb was provided with a primer consisting in a thermal profile characterized by a central peak temperature followed by a constant negative thermal gradient up to a lagrangian mass coordinate $M_{\text {pri }}$ where the temperature went down to $10^{8} \mathrm{~K}$, and remained constant at this value for the remaining of the ball. The numerical setup of these numerical experiments, as well as the physics included, were alike those described in Niemeyer \& Woosley [1997]. Once the sphere was allowed to evolve it was subject to two opposite competing effects: on the one hand it expanded and cooled due to the excess pressure exerted by the sudden incineration of the hot primer but, on the other hand, ongoing reactions released nuclear energy that helped keeping a high value of temperature. Which of the two effects wins is what determines if a detonation is successfully launched or not. The numerical experiments are therefore characterized by four parameters: the central temperature, $T_{\mathrm{c}}$, the uniform sphere density, $\rho$, the mass of the primer, $M_{\mathrm{pri}}$, and the $\mathrm{He}$ mass fraction, $X(\mathrm{He})$. In order to limit the dimensionality of the problem we have performed the calculations at a fixed density: $\rho=1.5 \times 10^{6} \mathrm{~g} \mathrm{~cm}^{-3}$, while a range has been explored for the rest of the parameters: $1.0 \times 10^{9} \mathrm{~K} \leqslant T_{\mathrm{c}} \leqslant 1.8 \times 10^{9} \mathrm{~K}, 3 \times 10^{25} \mathrm{~g} \leqslant M_{\text {pri }} \leqslant 3 \times 10^{29} \mathrm{~g}, 0.02 \leqslant$ $X(\mathrm{He}) \leqslant 0.20$. For the extreme values of primer mass considered the ball sound crossing time ranges from $0.01 \mathrm{~s}\left(M_{\text {pri }}=3 \times 10^{25} \mathrm{~g}\right)$ to $0.3 \mathrm{~s}\left(M_{\text {pri }}=3 \times 10^{29} \mathrm{~g}\right)$, meaning that as the primer mass increases there is more time for building the detonation structure and hence it is easier to start such a detonation. The detonation initiation conditions in pure $\mathrm{C}-\mathrm{O}$ matter (50\% each by mass) are given in Table 1, while our results concerning the detonation initiation conditions in He-contaminated 


\begin{tabular}{rlc}
\hline $\begin{array}{r}\rho \\
\left(\mathrm{g} \mathrm{cm}^{-3}\right)\end{array}$ & $\begin{array}{l}T_{\mathrm{C}} \\
\left(10^{9} \mathrm{~K}\right)\end{array}$ & $\begin{array}{c}M_{\mathrm{pri}}^{a} \\
(\mathrm{~g})\end{array}$ \\
\hline $3 \times 10^{6}$ & 2.3 & $2 \times 10^{28}$ \\
$10^{7}$ & 1.9 & $1.5 \times 10^{27}$ \\
$10^{7}$ & 2 & $1.1 \times 10^{24}$ \\
$10^{7}$ & 2.2 & $2 \times 10^{25}$ \\
$10^{7}$ & 2.8 & $2.5 \times 10^{23}$ \\
$10^{7}$ & 3.5 & $2 \times 10^{23}$ \\
$2 \times 10^{7}$ & 2 & $2.9 \times 10^{21}$ \\
$3 \times 10^{7}$ & 2 & $4.9 \times 10^{20}$ \\
$3 \times 10^{7}$ & 5.2 & $2 \times 10^{19}$ \\
$5 \times 10^{7}$ & 2 & $1.1 \times 10^{19}$ \\
$10^{8}$ & 2 & $5.5 \times 10^{17}$ \\
$10^{8}$ & 6.2 & $2 \times 10^{15}$ \\
\hline
\end{tabular}

(a) Compiled from Arnett \& Livne [1994], Niemeyer \& Woosley [1997] and Röpke et al. [2007]

Table 1: Summary of detonation initiation conditions in C-O matter

C-O matter are shown in Table 2.

The data in Table 1 can be interpreted either as the minimum primer mass for which a detonation is initiated in $\mathrm{C}-\mathrm{O}$ matter at a given density and peak temperature or as the minimum temperature for which a detonation is correspondingly obtained at a given density and mass of the primer. Either way, the qualitative trend is that the smaller the density the harder to initiate a detonation while, at fixed density, the higher the peak temperature the less primer mass is needed to detonate the sphere. From Table 1 it stems that at densities in the range $10^{7}-10^{8} \mathrm{~g} \mathrm{~cm}^{-3}$ the absolute minimum temperature needed to initiate a detonation is $\sim 2 \times 10^{9} \mathrm{~K}$. At lower densities (e.g. $3 \times 10^{6} \mathrm{~g} \mathrm{~cm}^{-3}$ the primer mass necessary to initiate a detonation at such temperatures becomes a non-negligible fraction of the star mass, hence achievement of the detonation initiation conditions becomes increasingly difficult.

As shown in Table 2 addition of a small quantity of He to the C-O sphere makes detonation initiation far easier. In our numerical experiments we have considered two distributions of $\mathrm{He}$ within the sphere, either constrained to the primer (in this case the role of $\mathrm{He}$ is limited to a larger release of nuclear energy with respect to pure $\mathrm{C}-\mathrm{O}$ ) or uniformly distributed through the sphere (thus taking also into account the larger reactivity of $\mathrm{He}$ with respect to $\mathrm{C}-\mathrm{O}$ matter). For a $10 \%$ He mass fraction a detonation is feasible even at a temperature as small as $10^{9} \mathrm{~K}$ provided that a large enough primer mass $\left(>1.5 \times 10^{-4} \mathrm{M}_{\odot}\right)$ is raised. Note that in this case the total mass of He needed is just above $1.5 \times 10^{-5} \mathrm{M}_{\odot}$. For a $5 \%$ He mass fraction the minimum temperature needed to achieve a stable detonation rises to $1.8 \times 10^{9} \mathrm{~K}$, for the same primer mass as before. The results obtained with a peak temperature of $1.4 \times 10^{9} \mathrm{~K}$ and $M_{\text {pri }}=3 \times 10^{27} \mathrm{~g}$ show an interesting behavior: a $20 \%$ mass fraction of He constrained to the primer was not able to initiate a detonation, while with a modest $10 \%$ He mass fraction uniformly distributed through the whole sphere a detonation 


\begin{tabular}{lcccc}
\hline $\begin{array}{l}T \\
\left(10^{9} \mathrm{~K}\right)\end{array}$ & $\begin{array}{c}M_{\mathrm{pri}}^{a} \\
(\mathrm{~g})\end{array}$ & $X(\mathrm{He})^{b}$ & He-distribution $^{c}$ & Detonation? \\
\hline 1.0 & $3 \times 10^{29}$ & 0.10 & primer & $\mathrm{y}$ \\
1.4 & $3 \times 10^{25}$ & 0.10 & unif. & $\mathrm{y}$ \\
1.4 & $3 \times 10^{27}$ & 0.02 & primer & $\mathrm{n}$ \\
1.4 & $3 \times 10^{27}$ & 0.05 & primer & $\mathrm{n}$ \\
1.4 & $3 \times 10^{27}$ & 0.10 & primer & $\mathrm{n}$ \\
1.4 & $3 \times 10^{27}$ & 0.20 & primer & $\mathrm{n}$ \\
1.4 & $3 \times 10^{27}$ & 0.10 & unif. & $\mathrm{y}$ \\
1.4 & $3 \times 10^{29}$ & 0.05 & primer & $\mathrm{n}$ \\
1.4 & $3 \times 10^{29}$ & 0.10 & primer & $\mathrm{y}$ \\
1.8 & $3 \times 10^{27}$ & 0.05 & unif. & $\mathrm{n}$ \\
1.8 & $3 \times 10^{29}$ & 0.05 & primer & $\mathrm{y}$ \\
\hline
\end{tabular}

(a) In all the calculations the density was $1.5 \times 10^{6} \mathrm{~g} \mathrm{~cm}^{-3}$ uniform

(b) Local He mass fraction

(c) Primer: He concentrated in the primer. Unif.: He uniformly distributed through the whole sphere

Table 2: Detonation initiation in $\mathrm{C}-\mathrm{O}$ matter with traces of $\mathrm{He}$

was obtained. This results suggest that the main reason by which He facilitates the transition to a detonation in $\mathrm{C}-\mathrm{O}$ matter is because of its larger reactivity rather than because of its larger nuclear energy release.

To conclude this section we remark that the initiation of a detonation is a highly non-linear process in which a modest variation in the environmental conditions can have a large impact on the evolution. The possibility that $\mathrm{He}$ is present mixed with $\mathrm{C}-\mathrm{O}$ when a DDT is prone to occur is just one example.

\section{Delayed detonation simulations}

Given the scarcity of three-dimensional simulations of the delayed detonation scenario and the complexity of the problem, it is interesting to perform additional calculations that explore the range of initial conditions of the DDT. In this section we present new three-dimensional simulations of delayed detonations in white dwarfs aimed to test the sensitivity of the results to the spatial locations of the DDT. The simulations were performed with a Smoothed Particle Hydrodynamics supernova code described in Bravo \& García-Senz [2008].

All the delayed detonation models presented here start by a common deflagration phase. This phase begins when the first sparks ignite in the central regions of the white dwarf and ends when the first detonation wave appears. Both modes of burning propagation can coexist for a while during the detonation phase although the efficiency of the deflagration is far below that of the supersonic 
detonation. In our computations the deflagration algorithm was switched off once a DDT was artificially induced.

The initial conditions were that of an isothermal Chandrasekhar-mass white dwarf in mechanical equilibrium. At $t=0$ the particles contained in a central volume were instantaneously incinerated, from this time on the flame propagated at a prescribed subsonic velocity during the deflagration phase. During the first half second the flame front remained nearly spherical. Afterwards, the fingers characteristic of the Rayleigh-Taylor instability developed very quickly, which resulted in hot material rising and cool unburnt carbon and oxygen intruding towards the center. As the flame surface increased due to the hydrodynamic instabilities, the rate of released nuclear energy and the effective burning velocity also did. At $t=0.9 \mathrm{~s}$ the burning front displayed the mushroomlike shape characteristic of the non-linear Rayleigh-Taylor regime, encompassing a large fraction of the white dwarf mass. As a consequence the rate of nuclear energy generation went up steeply until a maximum value of $1.3 \times 10^{51} \mathrm{erg} \mathrm{s}^{-1}$ was achieved at $\mathrm{t}=1.1 \mathrm{~s}$. At $t=1.55 \mathrm{~s}$ the average flame density was on the order of $2 \times 10^{7} \mathrm{~g} \mathrm{~cm}^{-3}$, and the deflagration algorithm was inefficient to propagate the flame. We took this moment as the time of onset of the detonation.

To explore the sensitivity of the results to the location of the DDT we used alternative prescriptions to select the particles in which a detonation had to be induced:

- Core detonation. All flame particles whose radius were lower than $1.3 \times 10^{8} \mathrm{~cm}$ were instantaneously incinerated. The density in these regions was higher than $\sim 4 \times 10^{7} \mathrm{~g} \mathrm{~cm}^{-3}$.

- Mid-altitude detonation. In this variant all flame particles within radii $1.7 \times 10^{8} \mathrm{~cm}$ and $2.0 \times 10^{8} \mathrm{~cm}$ were instantaneously incinerated. Their mean density was approximately $\sim$ $2 \times 10^{7} \mathrm{~g} \mathrm{~cm}^{-3}$.

- Atmospheric detonation. All flame particles within $2.4 \times 10^{8} \mathrm{~cm}$ and $2.8 \times 10^{8} \mathrm{~cm}$ were instantaneously incinerated. Their densities ranged from $\sim 7 \times 10^{6} \mathrm{~g} \mathrm{~cm}^{-3}$ to $\sim 10^{7} \mathrm{~g} \mathrm{~cm}^{-3}$.

In either case, after a transient induction period, a stable detonation was formed. Because the detonation was initiated in a more or less uniform way in a large volume within the white dwarf, there followed a period of rapid and generalized combustion leading to a temperature distribution much more homogeneous than that of the preceding deflagration phase. There were several detonation fronts cut off from each other because of the presence of ashes from the previous deflagration phase. Those detonation fronts propagating close to the center synthesized additional quantities of ${ }^{56} \mathrm{Ni}$, whereas detonations traveling through lower density layers synthesized intermediate-mass elements. In this way, the DDT allowed the partial compensation of the shortcomings of the previous deflagration stage, giving an improved explosion model.

The results of the simulations are given in Table 3, together with the results from Gamezo et al. [2005] for comparison purposes. The total amount of ${ }^{56} \mathrm{Fe}$ (after radioactive decay) and intermediate-mass elements ejected in the explosion are compatible with, but on the low side of, the range allowed by SNIa observations. Another positive feature of the model is that the ashes are not concentrated in large clumps, contrary to what is usually obtained in pure deflagration models. In addition, there remains little unburnt carbon and oxygen close to the center. However, there are too many Fe-group elements close to the surface of the white dwarf, especially too much radioactive ${ }^{56} \mathrm{Ni}$. Moreover, the ejecta is not chemically stratified. Stable Fe-group nuclei are nearly absent 


\begin{tabular}{lccccccc}
\hline Model & $\begin{array}{c}K \\
\left(10^{51} \mathrm{erg}\right)\end{array}$ & $\begin{array}{c}M_{\mathrm{NSE}} \\
\left(M_{\odot}\right)\end{array}$ & $\begin{array}{c}M_{56} \\
\left(M_{\odot}\right)\end{array}$ & $\begin{array}{c}M_{\text {ime }} \\
\left(M_{\odot}\right)\end{array}$ & $\begin{array}{c}M_{\mathrm{CO}} \\
\left(M_{\odot}\right)\end{array}$ & $\begin{array}{c}M_{\max } \\
(\mathrm{mag})\end{array}$ & $\begin{array}{c}\Delta M_{15} \\
(\mathrm{mag})\end{array}$ \\
\hline Central DDT $^{a}$ & 0.8 & 0.78 & & & & & \\
Off-center DDT $^{a}$ & 0.8 & 0.73 & & & & & \\
Central high-density DDT $^{a}$ & 1.1 & 0.94 & & & & & \\
Core detonation $^{b}$ & 0.48 & 0.71 & 0.43 & 0.10 & 0.48 & -18.63 & 0.57 \\
Mid-altitude detonation $^{b}$ & 0.51 & 0.69 & 0.42 & 0.14 & 0.45 & -18.76 & 0.66 \\
Atmospheric detonation $^{b}$ & 0.33 & 0.59 & 0.34 & 0.09 & 0.57 & -18.54 & 0.95 \\
\hline
\end{tabular}

(a) Gamezo et al. [2005]

(b) This work

Table 3: Results of 3D delayed detonation simulations

from the low velocity layers of the ejecta, which does not match the requirements from infrared observations of SNIa (Motohara et al. [2006], Gerardy et al. [2007], Fesen et al.. [2007]).

Compared to the 3D DDT models calculated by Gamezo et al. [2005], our models kinetic energy is substantially lower, although the amount of Fe-group nuclei synthesized is similar. This points to a quite different production of intermediate-mass elements, as a result of a different time of induction of the DDT in both classes of models. In general, Gamezo et al. assume the DDT to occur at a higher density than in our models. With respect to the effect of the spatial location of the DDT, both Gamezo et al. models (central vs off-center) and our models show that the outcome of the explosion is not quite sensitive to it. The diversity of supernova explosions obtained with the different prescriptions used for the DDT is not as rich as that usually achieved in one-dimensional delayed detonations. The kinetic energy varies at most in 0.15 foes, whereas the range of ${ }^{56} \mathrm{Ni}$ masses spans from 0.34 to $0.43 \mathrm{M}_{\odot}$. By contrast, one-dimensional models with transition densities in the same range as we have explored produce explosions with kinetic energies that change by 0.34 foes and ${ }^{56} \mathrm{Ni}$ masses ranging from 0.38 to $0.97 \mathrm{M}_{\odot}$ (Badenes et al. [2003, 2005]).

In Table 3 we give as well the results of the calculations of bolometric light curves of our 3D delayed detonation models. These light curve calculations were performed in one dimension, assuming spherical symmetry, from angle-averaged versions of the 3D explosion models. The method followed to calculate the light curve was the same as in Bravo et al. [1993]. The last two columns of the table give the maximum bolometric magnitude and the decline in bolometric magnitude during the first 15 days following the maximum, $\Delta M_{15}$. The maximum luminosities achieved by our models are small compared with normal bright SNIa, but are still comparable to normal or peculiar underluminous SNIa. Such a comparison is performed in Fig. 1, where we plot the relationship between the mass of ${ }^{56} \mathrm{Ni}$ synthesized and $\Delta M_{15}$, together with observational data obtained by Stritzinger et al. [2006]. The atmospheric detonation model fit nicely with the observational data, whereas the light curve of the central and mid-altitude detonations are too broad ${ }^{1}$.

\footnotetext{
${ }^{1}$ The most energetic 3D deflagration models are able to fit as well as the atmospheric detonation model, see Röpke et al. [2007]
} 


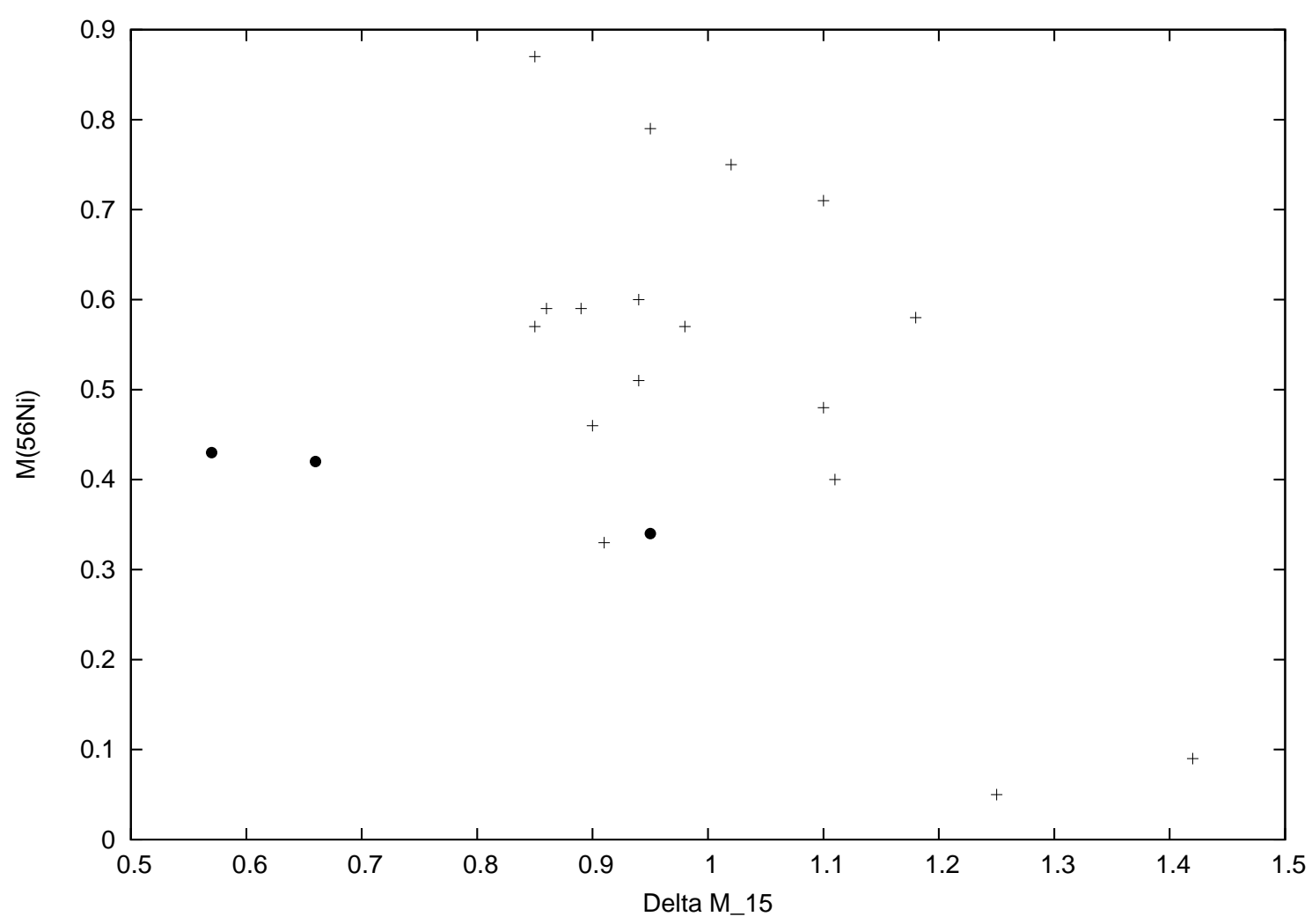

Figure 1: Relationship between the mass of ${ }^{56} \mathrm{Ni}$ synthesized in SNIa explosions and the decrease in bolometric magnitude 15 days after maximum, $\Delta M_{15}$. Crosses are data for a sample of well observed SNIa from Stritzinger et al. [2006], circles belong to the present 3D delayed detonation models

With respect to the models from Gamezo et al. [2005] in Table 1, their larger ${ }^{56} \mathrm{Ni}$ mass and kinetic energy is expected to imply a faster decrease of the bolometric magnitude after maximum than our delayed detonation models, hence a smaller value of $\Delta M_{15}$ that would put them out of the trend of observational data in Fig. 1. However, in the absence of actual light curve calculations of their models, any conclusion about their predicted observational properties has to be considered as very speculative.

\section{Conclusions}

In spite of recent advances in 3D modeling of delayed detonations of white dwarfs, current models are still far from passing basic observational tests. The few one-dimensional spectral calculations from angle-averaged 3D SNIa models (e.g. Baron et al. [2008]) did isolate several deficiencies when compared to observed SNIa spectra: the formation of undetected lines of C, an incorrect shape of the $\mathrm{S}$ lines, and an excessive abundance of Fe-group elements in the outer layers of the ejecta. On the other hand, observed bolometric light curves are easily reproduced by current 3D models of SNIa.

One of the most interesting questions concerning 3D SNIa models is the chemical profile of the ejecta. Observations suggest that supernova ejecta typically consist of a smooth, central, 
iron-rich core and an outer layer with chemical asymmetries (Gerardy et al. [2007], Wang, Baade \& Patat [2007], Mazzali et al. [2008]). The stratified core retains memory of the initial flame propagation, with electron-capture nuclear statistical equilibrium (NSE) elements at the center (innermost $0.2-0.3 \mathrm{M}_{\odot}$ ) and radioactive ${ }^{56} \mathrm{Ni}$ surrounding it. The outer layer is thoroughly mixed, dominated by intermediate-mass elements (mainly $\mathrm{Si}$ ) with a non-negligible abundance of oxygen and Fe-group elements. Such a chemical profile suggests the following properties of the explosion:

1. The deflagration front has to be stable at small scalelengths in order to avoid turbulent acceleration of the flame and allow substantial electron captures to proceed.

2. The deflagration front has to be stable at large scalelengths in order to avoid mixing out stable Fe-group nuclei.

3. A sudden, probably inhomogeneous, acceleration of the burning rate, following expansion by a factor 4-5 in radius, starts a detonation wave.

Matching these requirements with parameter-free 3D simulations of white dwarf thermonuclear explosions would be a high achievement of supernova theory.

\section{References}

[1994] D. Arnett, E. Livne, The delayed-detonation model of Type IA supernovae. 2: The detonation phase, ApJ 427330 (1994)

[2003] C. Badenes, E. Bravo, K.J. Borkowski, I. Domínguez, Thermal X-ray emission from shocked ejecta in Type Ia supernova remnants: Prospects for explosion mechanism identification, ApJ 593358 (2003)

[2005] C. Badenes, K.J. Borkowski, E. Bravo, J.P. Hughes, U. Hwang, Exploring the physics of Type Ia supernovae through the X-ray spectra of their remnants, Memorie della Societa Astronomica Italiana 76555 (2005)

[2006] C. Badenes, K.J. Borkowski, J.P. Hughes, U. Hwang, E. Bravo, Constraints on the physics of Type Ia supernovae from the X-ray spectrum of the Tycho supernova remnant, ApJ 6451373 (2006)

[2003] E. Baron, E.J. Lentz, P.H. Hauschildt, Detectability of mixed unburnt $C+O$ in Type Ia supernova spectra, ApJ 588 L29 (2003)

[2008] E. Baron, D.J. Jeffery, D. Branch, E. Bravo, D. García-Senz, P.H. Hauschildt, Detailed spectral modeling of a three-dimensional pulsating reverse detonation model: Too much nickel, ApJ 6721038 (2008)

[1993] E. Bravo, I. Domínguez, J. Isern, R. Canal, P. Höflich, J. Labay, On the photometric homogeneity of Type Ia supernovae, A\& A 269187 (1993)

[2006] E. Bravo, D. García-Senz, Beyond the bubble catastrophe of Type Ia supernovae: Pulsating reverse detonation models, ApJ 642 L157 (2006)

[2008] E. Bravo, D. García-Senz, A three-dimensional picture of the delayed-detonation model of type Ia supernovae, A\& A $\mathbf{4 7 8} 843$ (2008)

[2008] D.A. Chamulak, E.F. Brown, F.X. Timmes, K. Dupczak, The reduction of the electron abundance during the pre-explosion simmering in white dwarf supernovae, ApJ 677160 (2008) 
[1994a] R.J. Cumming, et al., Supernova 1994D in NGC 4526, IAU Circ. 5951 (1994)

[1994b] R.J. Cumming, W.P.S. Meikle, T.R. Geballe, R. Royer, G.M. Hurst, G. Comello, W.G. Dillon, Supernova 1994D in NGC 4526, IAU Circ. 5953 (1994)

[2007] R.A. Fesen, et al. The chemical distribution in a subluminous Type Ia supernova: Hubble Space Telescope images of the SN 1885 remnant, ApJ $\mathbf{6 5 8} 396$ (2007)

[2004] V.N. Gamezo, A.M. Khokhlov, E.S. Oran, Deflagrations and detonations in thermonuclear supernovae, PRL 921102 (2004)

[2005] V.N. Gamezo, A.M. Khokhlov, E.S. Oran, Three-dimensional Delayed-Detonation Model of Type Ia Supernovae, ApJ 623337 (2005)

[2003] D. García-Senz, E. Bravo, Nucleosynthesis as a result of multiple delayed detonations in type Ia supernovae. Nuc.Phys.A 718563 (2003)

[2007] D. García-Senz, E. Bravo, R.M. Cabezón, S.E. Woosley, Constraining deflagration models of Type Ia supernovae through intermediate-mass elements, ApJ 660509 (2007)

[2007] C.L. Gerardy, et al., Signatures of delayed detonation, asymmetry, and electron capture in the mid-infrared spectra of supernovae 2003hv and 2005df, ApJ 661995 (2007)

[2005] I. Golombek, J.C. Niemeyer, A model for multidimensional delayed detonations in SN Ia explosions, A\&A 438611 (2005)

[1999] K. Hatano, D. Branch, A. Fisher, E. Baron, A.V. Filippenko, On the high-velocity ejecta of the Type Ia supernova SN 1994D, ApJ 525881 (1999)

[2000] K. Hatano, D. Branch, E.J. Lentz, E. Baron, A.V. Filippenko, P.M. Garnavich, On the spectroscopic diversity of Type Ia supernovae, ApJ 543 L49 (2000)

[2000] W. Hillebrandt, J.C. Niemeyer, Type Ia Supernova Explosion Models, Ann. Rev. Astron. Astroph. 38 $191(2000)$

[1996] P. Höflich, A. Khokhlov, Explosion models for Type Ia supernovae: A comparison with observed light curves, distances, HO, and QO, ApJ 457500 (1996)

[1974] L.N. Ivanova, V.S. Imshennik, V.M. Chechetkin, Pulsation regime of the thermonuclear explosion of a star's dense carbon core, Astrophysics \& Space Science 31497 (1974)

[1991] A. Khokhlov, Delayed detonation model for type Ia supernovae, A\&A 245114 (1991)

[1997] A.M. Khokhlov, E.S. Oran, J.C. Wheeler, Deflagration-to-detonation transition in thermonuclear supernovae, ApJ 478678 (1997)

[2005] C. Kozma, et al., Three-dimensional modeling of type Ia supernovae - The power of late time spectra, A\&A 437983 (2005)

[2006] M. Kuhlen, S.W. Woosley, G.A. Glatzmaier, Carbon ignition in Type Ia supernovae. II. A three-dimensional numerical model, ApJ 640407 (2006)

[2000] A.M. Lisewski, W. Hillebrandt, S.E. Woosley, Constraints on the delayed transition to detonation in Type Ia supernovae, ApJ 538831 (2000)

[1999] E. Livne, Delayed detonation at a single point in exploding white dwarfs, ApJ 527 L97 (1999)

[2003] G.H. Marion, P. Höflich, W.D. Vacca, J.C. Wheeler, Near-infrared spectra of Type Ia supernovae, ApJ 591316 (2003) 
[1998] P.A. Mazzali, L.B. Lucy, The 1.05- $m$ feature in the spectrum of the Type Ia supernova 1994D: He in SNe Ia?, MNRAS 295428 (1998)

[2007] P.A. Mazzali, F.K. Röpke, S. Benetti, W. Hillebrndt, A common explosion mechanism for Type Ia supernovae, Science 315825 (2007)

[2008] P.A. Mazzali, D.N. Sauer, A. Pastorello, S. Benetti, W. Hillebrandt, Abundance stratification in Type Ia supernovae - II: The rapidly declining, spectroscopically normal, SN 2004 eo, MNRAS in press (2008)

[2006] K. Motohara, et al. The asymmetric explosion of Type Ia supernovae as seen from near-infrared observations, ApJ 652 L101 (2006)

[2002] R. Napiwotzki, et al. (SPY consortium), Binaries discovered by the SPY project. II. HE 1414-0848: A double degenerate with a mass close to the Chandrasekhar limit, A\&A 386957 (2002)

[1999] J.C. Niemeyer, Can deflagration-detonation transitions occur in Type Ia supernovae?, ApJ 523 L57 (1999)

[1997] J.C. Niemeyer, S.E. Woosley, The Thermonuclear Explosion of Chandrasekhar Mass White Dwarfs, ApJ 475740 (1997)

[2003] K. Nomoto, T. Uenishi, C. Kobayashi, H. Umeda, T. Ohkubo, I. Hachisu, M. Kato, Type Ia supernovae: Progenitors and diversities, in proceedings of ESO/MPA/MPE Workshop From twilight to highlight: The physics of supernovae, ed. W. Hillebrandt, B. Leibundgut (Springer-Verlag), 115 (2003)

[1997] P. Nugent, E. Baron, P. Hauschildt, D. Branch, Synthetic spectra of hydrodynamic models of Type Ia supernovae, ApJ 485812 (1997)

[2008] G. Pignata, et al., Optical and IR observations of SN 2002dj: Some possible common properties of fast expanding SNe Ia, MNRAS, in press (2008)

[2008] A.L. Piro, L. Bildsten, Neutronization during Type Ia supernova simmering, ApJ 6731009 (2008)

[2004] T. Plewa, A.C. Calder, D.Q. Lamb, Type Ia Supernova Explosion: Gravitationally Confined Detonation, ApJ 612 L37 (2004)

[2004] F.K. Röpke, W. Hillebrandt, J.C. Niemeyer, The cellular burning regime in type Ia supernova explosions. II. Flame propagation into vortical fuel, A\&A 421783 (2004)

[2007a] F.K. Röpke, J.C. Niemeyer, Delayed detonations in full-star models of type Ia supernova explosions, A\&A 464683 (2007)

[2007b] F.K. Röpke, S.E. Woosley, W. Hillebrandt, Off-center ignition in Type Ia supernovae. I. Initial evolution and implications for delayed detonation, ApJ 6601344 (2007)

[2007c] F.K. RÃúpke, W. Hillebrandt, W. Schmidt, J.C. Niemeyer, S.I. Blinnikov, P.A. Mazzali, $A$ three-dimensional deflagration model for Type Ia supernovae compared with observations, ApJ 668 1132 (2007)

[1998] H. Saio, K. Nomoto, Inward propagation of nuclear-burning shells in merging C-O and He white dwarfs, ApJ $\mathbf{5 0 0} 388$ (1998)

[1997] L. Segretain, G. Chabrier, R. Mochkovitch, The fate of merging white dwarfs, ApJ 481355 (1997)

[2006] M. Stritzinger, P.A. Mazzali, J. Sollerman, S. Benetti, Consistent estimates of 56Ni yields for type Ia supernovae, A\& A 460793 (2006) 
[2002] R.C. Thomas, D. Kasen, D. Branch, E. Baron, Spectral consequences of deviation from spherical composition symmetry in Type Ia supernovae, ApJ 5671037 (2002)

[1992] F.X. Timmes, S.E. Woosley, The conductive propagation of nuclear flames. I - Degenerate $C+O$ and $O+N E+M G$ white dwarfs, ApJ 396649 (1992)

[2007] L. Wang, D. Baade, F. Patat, Spectropolarimetric diagnostics of thermonuclear supernova explosions, Science 315212 (2007)

[1998] J.C. Wheeler, P. Höiň́Ćich, R.P. Harkness, J. Spyromilio, Explosion diagnostics of Type Ia supernovae from early infrared spectra, ApJ 496908 (1998)

[2007] M. Zingale, L.J. Dursi, Propagation of the first flames in Type Ia supernovae, ApJ 656333 (2007) 